\title{
Conditional Oscillation of Half-Linear Differential Equations with Coefficients Having Mean Values
}

\author{
Petr Hasil, ${ }^{1}$ Robert Mařík, ${ }^{2}$ and Michal Veselý ${ }^{1}$ \\ ${ }^{1}$ Department of Mathematics and Statistics, Faculty of Science, Masaryk University, Kotlárská 2, 61137 Brno, Czech Republic \\ ${ }^{2}$ Department of Mathematics, Faculty of Forestry and Wood Technology, Mendel University in Brno, Zemědělská 1, \\ 61300 Brno, Czech Republic
}

Correspondence should be addressed to Michal Veselý; michal.vesely@mail.muni.cz

Received 3 February 2014; Accepted 12 June 2014; Published 8 July 2014

Academic Editor: Yuriy Rogovchenko

Copyright (C) 2014 Petr Hasil et al. This is an open access article distributed under the Creative Commons Attribution License, which permits unrestricted use, distribution, and reproduction in any medium, provided the original work is properly cited.

\begin{abstract}
We prove that the existence of the mean values of coefficients is sufficient for second-order half-linear Euler-type differential equations to be conditionally oscillatory. We explicitly find an oscillation constant even for the considered equations whose coefficients can change sign. Our results cover known results concerning periodic and almost periodic positive coefficients and extend them to larger classes of equations. We give examples and corollaries which illustrate cases that our results solve. We also mention an application of the presented results in the theory of partial differential equations.
\end{abstract}

\section{Introduction}

In this paper, we analyse oscillatory properties of the halflinear differential equation

$$
\left[r(t) \Phi\left(x^{\prime}\right)\right]^{\prime}+z(t) \Phi(x)=0, \quad \Phi(x)=|x|^{p-1} \operatorname{sgn} x,
$$

where $r$ and $z$ are continuous functions, $r$ is positive, and $p>1$. To describe our main interest, let us consider (1) with $z(t)=\gamma s(t) t^{-p}$ for a continuous function $s$ and $\gamma \in \mathbb{R}$. We say that such an equation is conditionally oscillatory if there exists the so-called oscillation constant $\Gamma \in \mathbb{R}$ such that the equation under consideration is oscillatory for $\gamma>\Gamma$ and nonoscillatory for $\gamma<\Gamma$. In fact, the oscillation constant depends on coefficients $r$ and $s$.

Looking back to the history (according to our best knowledge), the first attempt to this problem was made by Kneser in [1], where the oscillation constant for the linear equation

$$
x^{\prime \prime}+\frac{\gamma}{t^{2}} x=0
$$

has been identified as $\Gamma=1 / 4$. Later, in $[2,3]$, it has been shown that the conditional oscillation remains preserved also for periodic coefficients. More precisely, the equation

$$
\left(r(t) x^{\prime}\right)^{\prime}+\gamma \frac{s(t)}{t^{2}} x=0
$$

where $r, s$ are positive $\alpha$-periodic continuous functions, is conditionally oscillatory for

$$
\Gamma=\frac{\alpha^{2}}{4}\left(\int_{0}^{\alpha} \frac{\mathrm{d} \tau}{r(\tau)}\right)^{-1}\left(\int_{0}^{\alpha} s(\tau) \mathrm{d} \tau\right)^{-1} .
$$

We also refer to more general results in [4-8].

Since a lot of results from the linear oscillation theory are extendable to the half-linear case (see, e.g., $[9,10])$, it is reasonable to suppose that the oscillation constant can be found for the corresponding Euler-type half-linear equations as well. This hypothesis has been shown to be true for

$$
\left[\Phi\left(x^{\prime}\right)\right]^{\prime}+\frac{\gamma}{t^{p}} \Phi(x)=0, \quad \Gamma=\left(\frac{p-1}{p}\right)^{p}
$$

in [11] (see also [12]). Later, this result has been extended in a number of papers (e.g., [13-17]), where equations of the below 
given form (6) have been treated with coefficients $r, s$ replaced by perturbations consisting of constant or periodic functions and iterated logarithms. Nevertheless, the most general result (concerning the topic of this paper) can be found in [18], where the equation

$$
\left[r(t) \Phi\left(x^{\prime}\right)\right]^{\prime}+\gamma \frac{s(t)}{t^{p}} \Phi(x)=0
$$

is treated for positive asymptotically almost periodic functions $r$ and $s$ satisfying

$$
\inf _{t \geq 0} r(t)>0, \quad \limsup _{t \rightarrow \infty} s(t)>0 .
$$

It is shown that (6) is conditionally oscillatory with the oscillation constant

$$
\Gamma=\left(\frac{p-1}{p}\right)^{p}\left[M\left(r^{1-q}\right)\right]^{1-p}[M(s)]^{-1},
$$

where $M(f)$ stands for the mean value of function $f$. This result is the main motivation of our current research. Our goal is to remove the condition of positivity of function $s$ and, at the same time, to extend the class of functions $r, s$ as much as possible applying the used methods. We present an oscillation criterion which is new in the half-linear case as well as in the linear one.

We should mention some relevant references from the discrete and time scale theory. In this paper, we give only the most relevant references concerning the topic. The reader can find more comprehensive literature overview together with historical references in our previous article [18]. Here, we refer at least to $[19,20]$ for the corresponding results about difference equations (see also, e.g., $[21,22]$ ) and to [23-25] for results about dynamical equations on time scales.

The paper is organized as follows. In the next section, we mention the necessary background and we recall the basics of the Riccati technique. In Section 3, we prove preparatory lemmas and our results. We also state several corollaries, concluding remarks, and examples. In the last section, we give an application in the theory of partial differential equations.

\section{Preliminaries}

Let $p>1$ be arbitrarily given and let $q>1$ be the real number conjugated with $p$ satisfying

$$
\frac{1}{p}+\frac{1}{q}=1, \text { i.e., } p+q=p q \text {. }
$$

As usual, for given $a>0$, the symbol $\mathbb{R}_{a}$ stands for $[a, \infty)$.

To prove the main results, we will apply the Riccati technique for (1), where the transformation

$$
w(t)=r(t) \Phi\left(\frac{x^{\prime}(t)}{x(t)}\right)
$$

leads to the half-linear Riccati differential equation

$$
w^{\prime}(t)+z(t)+(p-1) r^{1-q}(t)|w(t)|^{q}=0
$$

whenever $x(t) \neq 0$. For details, we refer to [10]. The fundamental connection between the nonoscillation of (1) and the solvability of (11) is described by the following theorem.
Theorem 1. Equation (1) is nonoscillatory if and only if there exists a function $w$ which solves (11) on some interval $[T, \infty)$.

Proof. The theorem is a consequence of the well-known roundabout theorem (see, e.g., [10, Theorem 1.2.2]).

We will also use the Sturmian comparison theorem in the form given below.

Theorem 2. Let $z, Z: \mathbb{R}_{a} \rightarrow \mathbb{R}$ be continuous functions satisfying $Z(t) \geq z(t)$ for all sufficiently larget. Let one consider (1) and the equation

$$
\left[r(t) \Phi\left(x^{\prime}\right)\right]^{\prime}+Z(t) \Phi(x)=0 .
$$

(i) If (1) is oscillatory, then (12) is oscillatory as well.

(ii) If (12) is nonoscillatory, then (1) is nonoscillatory as well.

Proof. The theorem follows, for example, from [10, Theorem 1.2.4].

Now we recall the concept of mean values which is necessary to find an explicit oscillation constant for general halflinear equations.

Definition 3. Let continuous function $f: \mathbb{R}_{a} \rightarrow \mathbb{R}$ be such that the limit

$$
M(f):=\lim _{t \rightarrow \infty} \frac{1}{t} \int_{b}^{b+t} f(s) \mathrm{d} s
$$

is finite and exists uniformly with respect to $b \in \mathbb{R}_{a}$. The number $M(f)$ is called the mean value of $f$.

In fact, we will study (1) in the form

$$
\begin{array}{r}
{\left[r^{-p / q}(t) \Phi\left(x^{\prime}\right)\right]^{\prime}+\frac{s(t)}{t^{p}} \Phi(x)=0,} \\
\Phi(x)=|x|^{p-1} \operatorname{sgn} x
\end{array}
$$

where $r: \mathbb{R}_{a} \rightarrow \mathbb{R}$ is a continuous function having mean value $M(r)=1$ and satisfying

$$
0<r^{-}:=\inf _{t \in \mathbb{R}_{a}} r(t) \leq r^{+}:=\sup _{t \in \mathbb{R}_{a}} r(t)<\infty
$$

and $s: \mathbb{R}_{a} \rightarrow \mathbb{R}$ is a continuous function having mean value $M(s)>0$. We repeat that the basic motivation comes from [18], where asymptotically almost periodic half-linear equations are analysed. Since positive nonvanishing asymptotically almost periodic functions have positive mean values and they are bounded, we will consider more general equations (cf. (15) with (7) as well). (11))

The Riccati equation associated to (14) has the form (see

$$
w^{\prime}(t)+\frac{s(t)}{t^{p}}+(p-1) r(t)|w(t)|^{q}=0 .
$$


Finally, using the substitution $w(t)=-\zeta(t) t^{1-p}$, we obtain the adapted Riccati equation

$$
\zeta^{\prime}(t)=\frac{1}{t}\left[(p-1) \zeta(t)+s(t)+(p-1) r(t)|\zeta(t)|^{q}\right],
$$

which will play a crucial role in the proof of the announced result (see the below given Theorem 8 ).

\section{Results}

To prove the announced result, we need the following lemmas.

Lemma 4. If there exists a solution of (17) on some interval $[T, \infty)$, then (14) is nonoscillatory.

Proof. A solution $\zeta$ of (17) on an interval $[T, \infty)$ gives the solution $w(t)=-\zeta(t) t^{1-p}$ of (16) on the same interval. Thus, the lemma follows from Theorem 1 .

Lemma 5. Let (14) be nonoscillatory and let there exist $M>0$ such that

$$
\left|\int_{b}^{c} \frac{s(\tau)}{\tau^{p}} d \tau\right|<M, \quad a \leq b<c \leq \infty .
$$

For any solution $w$ of $(16)$ on $[T, \infty)$, it holds

$$
\int_{T}^{\infty} r(\tau)|w(\tau)|^{q} d \tau<\infty
$$

Proof. The lemma follows, for example, from [10, Theorem 2.2.3], where it suffices to use (15).

Lemma 6. If (14) is nonoscillatory, then there exists a solution $\zeta$ of $(17)$ on some interval $[T, \infty)$ with the property that $\zeta(t)<$ $A$ for all $t \geq T$ and for some $A>0$.

Proof. Considering Theorem 1, the nonoscillation of (14) implies that there exists a solution $w$ of (16) on some interval $[T, \infty)$ which gives the solution $\zeta(t)=-w(t) t^{p-1}$ of $(17)$ on the interval. We show that this solution $\zeta$ is bounded above.

At first, we prove the convergence of the integral

$$
\int_{T}^{\infty} \frac{s(\tau)}{\tau^{p}} \mathrm{~d} \tau \in \mathbb{R}
$$

and the inequality

$$
\sup _{t \geq T}\left|t^{p-1} \int_{t}^{\infty} \frac{s(\tau)}{\tau^{p}} \mathrm{~d} \tau\right|<L \quad \text { for some } L>0 .
$$

Evidently, it suffices to prove (20) and

$$
\limsup _{t \rightarrow \infty}\left|t^{p-1} \int_{t}^{\infty} \frac{s(\tau)}{\tau^{p}} \mathrm{~d} \tau\right|<\infty .
$$

Let $b>0$ be such that

$$
\int_{t}^{t+b} s(\tau) \mathrm{d} \tau>0, \quad\left|\int_{t}^{t+b} s(\tau) \mathrm{d} \tau-b M(s)\right|<b, \quad t \geq T,
$$

where we use directly Definition 3 (the existence of $M(s)>$ $0)$. The symbols $[f(\cdot)]^{+}$and $[f(\cdot)]^{-}$will denote the positive and negative parts of function $f$, respectively. We choose $t_{0} \geq$ $T$. We can express

$$
\begin{aligned}
\int_{t_{0}+(k-1) b}^{t_{0}+k b} \frac{s(\tau)}{\tau^{p}} \mathrm{~d} \tau= & \int_{t_{0}+(k-1) b}^{t_{0}+k b} \frac{[s(\tau)]^{+}}{\tau^{p}} \mathrm{~d} \tau \\
& -\int_{t_{0}+(k-1) b}^{t_{0}+k b} \frac{[s(\tau)]^{-}}{\tau^{p}} \mathrm{~d} \tau, \quad k \in \mathbb{N} .
\end{aligned}
$$

For an arbitrarily given positive integer $k$, we have

$$
\begin{aligned}
I_{k}:= & \int_{t_{0}+(k-1) b}^{t_{0}+k b} \frac{s(\tau)}{\tau^{p}} \mathrm{~d} \tau \\
\leq & \frac{1}{\left[t_{0}+(k-1) b\right]^{p}} \int_{t_{0}+(k-1) b}^{t_{0}+k b}[s(\tau)]^{+} \mathrm{d} \tau \\
& -\frac{1}{\left[t_{0}+k b\right]^{p}} \int_{t_{0}+(k-1) b}^{t_{0}+k b}[s(\tau)]^{-} \mathrm{d} \tau
\end{aligned}
$$

if $I_{k}>0$, and

$$
\begin{aligned}
& -\int_{t_{0}+(k-1) b}^{t_{0}+k b} \frac{s(\tau)}{\tau^{p}} \mathrm{~d} \tau \\
& \leq-\frac{1}{\left[t_{0}+k b\right]^{p}} \int_{t_{0}+(k-1) b}^{t_{0}+k b}[s(\tau)]^{+} \mathrm{d} \tau \\
& \quad+\frac{1}{\left[t_{0}+(k-1) b\right]^{p}} \int_{t_{0}+(k-1) b}^{t_{0}+k b}[s(\tau)]^{-} \mathrm{d} \tau
\end{aligned}
$$

if $I_{k}<0$. Using

$$
\lim _{k \rightarrow \infty} \frac{\left[t_{0}+(k-1) b\right]^{p}}{\left[t_{0}+k b\right]^{p}}=1
$$

and using (23), (25), and (26), we obtain the existence of $n_{0} \in$ $\mathbb{N}$ such that it holds

$$
\left|\int_{t_{0}+(k-1) b}^{t_{0}+k b} \frac{s(\tau)}{\tau^{p}} \mathrm{~d} \tau\right|<\frac{2 b[M(s)+1]}{\left[t_{0}+(k-1) b\right]^{p}}, \quad k \geq n_{0}, k \in \mathbb{N} .
$$

Since $t_{0} \geq T$ is arbitrary, it also holds

$$
\left|\int_{t}^{t+b} \frac{s(\tau)}{\tau^{p}} \mathrm{~d} \tau\right|<\frac{2 b[M(s)+1]}{t^{p}}
$$

for all sufficiently large $t$.

Hence, the integral $\int_{T}^{\infty} s(\tau) \tau^{-p} \mathrm{~d} \tau$ is convergent because

$$
\begin{aligned}
\left|\int_{T}^{\infty} \frac{s(\tau)}{\tau^{p}} \mathrm{~d} \tau\right| & \leq\left|\int_{T}^{t} \frac{s(\tau)}{\tau^{p}} \mathrm{~d} \tau\right|+\left|\int_{t}^{\infty} \frac{s(\tau)}{\tau^{p}} \mathrm{~d} \tau\right| \\
& =\left|\int_{T}^{t} \frac{s(\tau)}{\tau^{p}} \mathrm{~d} \tau\right|+\left|\sum_{k=1}^{\infty}\left(\int_{t+(k-1) b}^{t+k b} \frac{s(\tau)}{\tau^{p}} \mathrm{~d} \tau\right)\right| \\
& \leq\left|\int_{T}^{t} \frac{s(\tau)}{\tau^{p}} \mathrm{~d} \tau\right|+\left|\sum_{k=1}^{\infty} \frac{2 b[M(s)+1]}{[t+(k-1) b]^{p}}\right|<\infty,
\end{aligned}
$$


where $t$ is sufficiently large. Particularly,

$$
\sup _{t \geq T}\left|\int_{T}^{t} \frac{s(\tau)}{\tau^{p}} \mathrm{~d} \tau\right|<K \quad \text { for some } K>0 .
$$

Moreover, we have (see (29))

$$
\begin{aligned}
& \limsup _{t \rightarrow \infty}\left|t^{p-1} \int_{t}^{\infty} \frac{s(\tau)}{\tau^{p}} \mathrm{~d} \tau\right| \\
& \quad=\limsup _{t \rightarrow \infty}\left|t^{p-1} \sum_{k=1}^{\infty}\left(\int_{t+(k-1) b}^{t+k b} \frac{s(\tau)}{\tau^{p}} \mathrm{~d} \tau\right)\right| \\
& \leq \limsup _{t \rightarrow \infty}\left|t^{p-1} \sum_{k=1}^{\infty} \frac{2 b[M(s)+1]}{[t+(k-1) b]^{p}}\right|<\infty .
\end{aligned}
$$

Thus, (22) is valid; that is, there exists $L>0$ for which (21) is valid.

Integrating (16), we obtain

$$
w(t)=w(T)-\int_{T}^{t} \frac{s(\tau)}{\tau^{p}} \mathrm{~d} \tau-(p-1) \int_{T}^{t} r(\tau)|w(\tau)|^{q} \mathrm{~d} \tau,
$$

We know that

$$
\begin{array}{r}
\int_{T}^{\infty} r(\tau)|w(\tau)|^{q} \mathrm{~d} \tau<\infty, \quad \text { i.e. }(\text { see }(15)), \\
\qquad \int_{T}^{\infty}|w(\tau)|^{q} \mathrm{~d} \tau<\infty .
\end{array}
$$

Indeed, considering (18) together with (31), one can get (19) from Lemma 5. From (20) and (33) it follows that there exists the limit $\lim _{t \rightarrow \infty} w(t) \in \mathbb{R}$. In addition, the convergence of the integral in (34) gives

$$
\lim _{t \rightarrow \infty}|w(t)|^{q}=0, \quad \text { i.e., } \lim _{t \rightarrow \infty} w(t)=0 \text {. }
$$

Again, we consider arbitrarily given $t_{0} \geq T$. We can rewrite (33) into (or see directly (16))

$$
\begin{array}{r}
w(t)=w\left(t_{0}\right)-\int_{t_{0}}^{t} \frac{s(\tau)}{\tau^{p}} \mathrm{~d} \tau-(p-1) \int_{t_{0}}^{t} r(\tau)|w(\tau)|^{q} \mathrm{~d} \tau \\
t \geq T .
\end{array}
$$

Putting $t_{0} \rightarrow \infty$, from (20), (34), (35), and (36), we obtain

$$
w(t)=\int_{t}^{\infty} \frac{s(\tau)}{\tau^{p}} \mathrm{~d} \tau+(p-1) \int_{t}^{\infty} r(\tau)|w(\tau)|^{q} \mathrm{~d} \tau, \quad t \geq T .
$$

Finally, let us denote $w(t)=f_{1}(t)+f_{2}(t)$, where

$$
\begin{aligned}
& f_{1}(t):=\int_{t}^{\infty} \frac{s(\tau)}{\tau^{p}} \mathrm{~d} \tau, \\
& f_{2}(t):=(p-1) \int_{t}^{\infty} r(\tau)|w(\tau)|^{q} \mathrm{~d} \tau, \quad t \geq T .
\end{aligned}
$$

We know that (see (21) and (37))

$$
\sup _{t \geq T} t^{p-1}\left|w(t)-f_{2}(t)\right|<L .
$$

We denote $S:=\{t \geq T: w(t)<0\}$. If $w$ is positive, then the statement of the lemma is true for all $A>0$. Therefore, we can assume that $S \neq \emptyset$. Since $f_{2}$ is nonincreasing and $\lim _{t \rightarrow \infty} f_{2}(t)=0$, function $f_{2}$ is nonnegative. From (39) it follows

$$
\sup _{t \in S} t^{p^{-1}}\left([w(t)]^{-}+f_{2}(t)\right)=\sup _{t \in S} t^{p^{-1}}\left|w(t)-f_{2}(t)\right|<L .
$$

Hence, we have

$$
\begin{aligned}
& \sup _{t \in S} t^{p-1}|w(t)| \\
& \quad=\sup _{t \in S} t^{p-1}\left([w(t)]^{-}+0\right) \leq \sup _{t \in S} t^{p-1}\left([w(t)]^{-}+f_{2}(t)\right)<L
\end{aligned}
$$

and, consequently, we obtain that $\zeta(t)=-t^{p-1} w(t)<L$, $t \geq T$. It means that the statement of the lemma is valid for $A=L$.

Remark 7. Let (14) be nonoscillatory. If the considered function $s$ is positive for all $t \geq a$, then the statement of Lemma 6 is true for a negative solution $\zeta$ of (17). See, for example, [10, Lemma 2.2.5].

Theorem 8. Equation (14) is oscillatory if $M(s)>q^{-p}$ and nonoscillatory if $M(s)<q^{-p}$.

Proof. The proof is organized as follows. In the first part, we derive upper bounds for two integrals involving function $s$. Then we prove the oscillatory part and, finally, the nonoscillatory part.

At first, we use the existence of $M(s)$ and the continuity of function $s$. Considering Definition 3, there exists $\beta \geq 1$ with the property that

$$
\begin{array}{r}
\left|\int_{b}^{b+\beta} s(\tau) \mathrm{d} \tau\right|<\beta[M(s)+1], \quad b \in[a, \infty), \\
\left|\frac{1}{\beta} \int_{b}^{b+\beta} s(\tau) \mathrm{d} \tau-\frac{1}{\beta+\xi} \int_{b}^{b+\beta+\xi} s(\tau) \mathrm{d} \tau\right|<1, \\
b \in[a, \infty), \quad \xi \in(0,1],
\end{array}
$$

and, consequently, there exists $R>0$ with the property that

$$
\left|\int_{c}^{c+\xi} s(\tau) \mathrm{d} \tau\right|<R, \quad c \in[a, a+\beta], \quad \xi \in(0,1] .
$$

We can rewrite (43) into the form

$$
\begin{array}{r}
\left|\frac{\xi}{\beta+\xi} \int_{b}^{b+\beta} s(\tau) \mathrm{d} \tau-\frac{\beta}{\beta+\xi} \int_{b+\beta}^{b+\beta+\xi} s(\tau) \mathrm{d} \tau\right|<\beta, \\
b \in[a, \infty), \quad \xi \in(0,1] .
\end{array}
$$


Using (42), we obtain

$$
\begin{aligned}
\left|\frac{\beta}{\beta+\xi} \int_{b+\beta}^{b+\beta+\xi} s(\tau) \mathrm{d} \tau\right| & <\beta+\frac{\xi}{\beta+\xi}\left|\int_{b}^{b+\beta} s(\tau) \mathrm{d} \tau\right| \\
& <\beta+\beta[M(s)+1] \\
b & \in[a, \infty), \quad \xi \in(0,1]
\end{aligned}
$$

that is,

$$
\left|\int_{b+\beta}^{b+\beta+\xi} s(\tau) \mathrm{d} \tau\right|<2 \beta[M(s)+2], \quad b \in[a, \infty), \xi \in(0,1] .
$$

Combining (44) and (47), we have

$$
\left|\int_{b}^{b+\xi} s(\tau) \mathrm{d} \tau\right|<S, \quad b \in[a, \infty), \quad \xi \in(0,1],
$$

where $S:=\max \{R, 2 \beta[M(s)+2]\}$. Since the function $y(t)=$ $1 / t$ is decreasing and positive on $\mathbb{R}_{a}$, it holds

$$
\int_{t_{1}}^{t_{2}} \frac{s(\tau)}{\tau} \mathrm{d} \tau=\frac{1}{t_{1}} \int_{t_{1}}^{t_{3}} s(\tau) \mathrm{d} \tau+\frac{1}{t_{2}} \int_{t_{3}}^{t_{2}} s(\tau) \mathrm{d} \tau
$$

for all $t_{2} \geq t_{1} \geq a$ and for some $t_{3} \in\left[t_{1}, t_{2}\right]$. Analogously, for any $t_{2} \geq t_{1} \geq a$, there exists $t_{4} \in\left[t_{1}, t_{2}\right]$ such that

$$
\int_{t_{1}}^{t_{2}} \frac{s(\tau)}{\tau} \mathrm{d} \tau=\frac{1}{t_{1}} \int_{t_{1}}^{t_{4}} s(\tau) \mathrm{d} \tau
$$

Hence, from (48) it follows

$$
\begin{array}{r}
\left|\int_{b}^{b+\xi} \frac{s(\tau)}{\tau} \mathrm{d} \tau\right| \leq \max _{\bar{\xi} \in[0, \xi]}\left|\int_{b}^{b+\bar{\xi}} \frac{s(\tau)}{b} \mathrm{~d} \tau\right|<\frac{S}{b}, \\
b \in[a, \infty), \quad \xi \in(0,1] .
\end{array}
$$

Now we prove the oscillatory part. Let $M(s)>q^{-p}$. By contradiction, in this part of the proof, we will suppose that (14) is nonoscillatory. Lemma 6 says that there exists a solution $\zeta$ of (17) on some interval $[T, \infty)$ and that $\zeta(t)<A$ for all $t \geq T$ and for a certain number $A>0$. Evidently, we can assume that $T>1$.

We show that there exists $K<-1$ satisfying

$$
\zeta(t)>K, \quad t \geq T \text {. }
$$

On the contrary, let us assume that $\liminf _{t \rightarrow \infty} \zeta(t)=-\infty$. Let $\zeta(t) \leq-P$ for all $t$ from some interval $\left[t_{1}, t_{2}\right]$, where $t_{2} \epsilon$ $\left(t_{1}+j-1, t_{1}+j\right]$ for some $j \in \mathbb{N}$, and let $P>0$ be such that (see (15))

$$
h(x):=(p-1) r^{-}|x|^{q}-S-(p-1)|x|>0, \quad|x| \geq P .
$$

Indeed, $\lim _{x \rightarrow \pm \infty} h(x)=\infty$. We can assume that $h$ is increasing for $x \geq P$. Using (17), (51), and (53), it holds

$$
\begin{aligned}
& \zeta\left(t_{2}\right)-\zeta\left(t_{1}\right) \\
& =\int_{t_{1}}^{t_{2}} \zeta^{\prime}(\tau) \mathrm{d} \tau \\
& =\int_{t_{1}}^{t_{2}} \frac{(p-1) \zeta(\tau)+s(\tau)+(p-1) r(\tau)|\zeta(\tau)|^{q}}{\tau} \mathrm{d} \tau \\
& \geq \int_{t_{1}}^{t_{2}} \frac{-(p-1) P+(p-1) r^{-} P^{q}}{\tau} \mathrm{d} \tau-\left|\int_{t_{1}}^{t_{2}} \frac{s(\tau)}{\tau} \mathrm{d} \tau\right| \\
& \geq \int_{t_{1}}^{t_{1}+1} \frac{-(p-1) P+(p-1) r^{-} P^{q}}{\tau} \mathrm{d} \tau-\left|\int_{t_{1}}^{t_{1}+1} \frac{s(\tau)}{\tau} \mathrm{d} \tau\right| \\
& +\cdots+\int_{t_{1}+j-2}^{t_{1}+j-1} \frac{-(p-1) P+(p-1) r^{-} P^{q}}{\tau} \mathrm{d} \tau \\
& -\left|\int_{t_{1}+j-2}^{t_{1}+j-1} \frac{s(\tau)}{\tau} \mathrm{d} \tau\right| \\
& +\int_{t_{1}+j-1}^{t_{2}} \frac{-(p-1) P+(p-1) r^{-} P^{q}}{\tau} \mathrm{d} \tau \\
& -\left|\int_{t_{1}+j-1}^{t_{2}} \frac{s(\tau)}{\tau} \mathrm{d} \tau\right| \\
& >\frac{S}{t_{1}+1}-\frac{S}{t_{1}}+\cdots+\frac{S}{t_{1}+j-1}-\frac{S}{t_{1}+j-2} \\
& +0-\frac{S}{t_{1}+j-1}=-\frac{S}{t_{1}} \text {. }
\end{aligned}
$$

Thus, $\zeta(t) \geq-P-S T^{-1}$ for all $t \geq T$ which proves (52). Indeed, it suffices to consider $\zeta\left(t_{1}\right)=-P$. In addition, we can assume that $-K>A$; that is,

$$
K<\zeta(t)<-K, \quad t \geq T .
$$

Thus (see directly (17) and (51)), we have

$$
\begin{aligned}
\left|\zeta\left(t_{2}\right)-\zeta\left(t_{1}\right)\right|= & \left|\int_{t_{1}}^{t_{2}} \zeta^{\prime}(\tau) \mathrm{d} \tau\right| \\
\leq & \int_{t_{1}}^{t_{2}} \frac{p-1}{\tau}\left[|\zeta(\tau)|+r(\tau)|\zeta(\tau)|^{q}\right] \mathrm{d} \tau \\
& +\left|\int_{t_{1}}^{t_{2}} \frac{s(\tau)}{\tau} \mathrm{d} \tau\right| \\
\leq & \frac{1}{t_{1}}\left[(p-1)|K|\left(t_{2}-t_{1}\right)+S\right. \\
& \left.+(p-1) r^{+}|K|^{q}\left(t_{2}-t_{1}\right)\right]
\end{aligned}
$$


for all $t_{1}, t_{2} \geq T$, where $t_{1}<t_{2} \leq t_{1}+1$. The previous inequality implies

$$
\begin{array}{r}
\left|\zeta\left(t_{2}\right)-\zeta\left(t_{1}\right)\right| \leq \frac{(p-1)|K|+S+(p-1) r^{+}|K|^{q}}{t_{1}}, \\
t_{1} \geq T, \quad t_{2} \in\left(t_{1}, t_{1}+1\right] .
\end{array}
$$

Considering Definition 3 and $M(s)>q^{-p}$, there exist $n \in$ $\mathbb{N}$ and $\varepsilon>0$ such that

$$
\frac{1}{n} \int_{b}^{b+n} s(\tau) \mathrm{d} \tau-q^{-p}>\varepsilon, \quad b \in[a, \infty),
$$

and, at the same time, such that

$$
1-\frac{\varepsilon}{4(p-1)|K|^{q}}<\frac{1}{n} \int_{b}^{b+n} r(\tau) \mathrm{d} \tau<2, \quad b \in[a, \infty) .
$$

For such an integer $n$, we define

$$
\vartheta(t):=\frac{1}{n} \int_{t}^{t+n} \zeta(\tau) \mathrm{d} \tau, \quad t \geq T .
$$

Since

$$
\frac{1}{n} \int_{t}^{t+n} \zeta(\tau) \mathrm{d} \tau<\frac{1}{n} \int_{t}^{t+n} A \mathrm{~d} \tau=A, \quad t \geq T,
$$

we have

$$
\vartheta(t)<A, \quad t \geq T .
$$

Hence, to prove the first implication in the statement of the theorem, it suffices to show that (62) is not true.

From (57) it follows

$$
|\zeta(t+\tau)-\zeta(t)| \leq \frac{C}{t}, \quad t \geq T, \tau \in[0, n],
$$

where

$$
C=n\left[(p-1)|K|+S+(p-1) r^{+}|K|^{q}\right] .
$$

Particularly (see (60)), (63) gives

$$
|\mathcal{V}(t)-\zeta(t)| \leq \max _{\tau \in[0, n]}|\zeta(t+\tau)-\zeta(t)| \leq \frac{C}{t}, \quad t \geq T .
$$

Next, we consider the function

$$
\begin{array}{r}
g_{1}(t):=(p-1) \zeta(t)+q^{-p}+\frac{p-1}{n} \int_{t}^{t+n} r(\tau)|\zeta(\tau)|^{q} \mathrm{~d} \tau, \\
t \geq T .
\end{array}
$$

If $\zeta(t) \geq 0$ for some $t \geq T$, then $g_{1}(t)>0$. Henceforth (in this paragraph), we consider the case when $\zeta(t)<0, t \geq T$. Let us define

$$
f(x):=x+\frac{q^{-p}}{p-1}+(-x)^{q}, \quad x \leq 0 .
$$

It can be directly verified that function $f$ has the global minimum

$$
\begin{aligned}
f\left(-q^{1 /(1-q)}\right) & \\
& =-q^{1 /(1-q)}+\frac{q^{-p}}{p-1}+q^{(1+q-1) /(1-q)} \\
& =q^{1 /(1-q)}\left(-1+q^{(q-1) /(1-q)}\right)+\frac{q^{-q /(q-1)}}{(q /(q-1))-1} \\
& =q^{1 /(1-q)}\left[\frac{1-q}{q}+\frac{q^{-1}}{(q-q+1) /(q-1)}\right] \\
& =q^{1 /(1-q)}\left[\frac{1-q}{q}+\frac{q-1}{q}\right]=0 .
\end{aligned}
$$

It means that $f(x) \geq 0, x \leq 0$. Particularly, it gives the inequality

$$
(p-1) \zeta(t)+q^{-p}+(p-1)|\zeta(t)|^{q} \geq 0, \quad t \geq T .
$$

Considering (59) and (69), we have

$$
\begin{aligned}
g_{2}(t) & :=(p-1) \zeta(t)+q^{-p}+\frac{p-1}{n} \int_{t}^{t+n} r(\tau)|\zeta(t)|^{q} \mathrm{~d} \tau \\
& \geq(p-1)|\zeta(t)|^{q}\left[\frac{1}{n} \int_{t}^{t+n} r(\tau) \mathrm{d} \tau-1\right] \\
& \geq(p-1)|\zeta(t)|^{q}\left[1-\frac{\varepsilon}{4(p-1)|K|^{q}}-1\right] \\
& \geq-\frac{\varepsilon}{4}, \quad t \geq T .
\end{aligned}
$$

Applying (63), the inequalities $K<\zeta(t)<A, t \geq T$, and the fact that the function $y=|x|^{q}$ has the Lipschitz property on any bounded set, there exists $\tilde{t} \geq T$ such that

$$
\left.|| \zeta(t)\right|^{q}-|\zeta(t+\tau)|^{q} \mid<\frac{\varepsilon}{8(p-1)}, \quad t \geq \tilde{t}, \tau \in[t, t+n] .
$$

Hence (see also (59)), we get

$$
\begin{aligned}
\mid g_{1}(t) & -g_{2}(t) \mid \\
& \leq\left.\frac{p-1}{n} \int_{t}^{t+n} r(\tau)|| \zeta(t)\right|^{q}-|\zeta(\tau)|^{q} \mid \mathrm{d} \tau \\
& \leq \frac{p-1}{n} \int_{t}^{t+n} r(\tau) \frac{\varepsilon}{8(p-1)} \mathrm{d} \tau<2(p-1) \frac{\varepsilon}{8(p-1)} \\
& =\frac{\varepsilon}{4}, \quad t \geq \tilde{t} .
\end{aligned}
$$


From (70) and (72), we know that

$$
g_{1}(t) \geq g_{2}(t)-\left|g_{2}(t)-g_{1}(t)\right| \geq-\frac{\varepsilon}{2}, \quad t \geq \tilde{t}
$$

Of course, (73) remains true for $\zeta(t) \geq 0$ as well.

Let us consider $\bar{t} \geq \tilde{t}$ for which

$$
|\zeta(t)-\vartheta(t)|<\frac{\varepsilon}{4(p-1)}, \quad t \geq \bar{t}
$$

Note that the existence of such a number $\bar{t}$ follows from (65). It is seen that (73) and (74) imply

$$
\begin{array}{r}
(p-1) \vartheta(t)+q^{-p}+\frac{p-1}{n} \int_{t}^{t+n} r(\tau)|\zeta(\tau)|^{q} \mathrm{~d} \tau \geq-\frac{3 \varepsilon}{4} \\
t \geq \bar{t}
\end{array}
$$

Evidently, we can consider the solution $\zeta$ in an arbitrarily given neighbourhood of $+\infty$. Hence, we can assume that

$$
\begin{gathered}
\frac{S n}{\bar{t}}<\frac{\varepsilon}{24}, \\
\frac{-K n(p-1)}{\bar{t}}<\frac{\varepsilon}{24}, \\
\frac{r^{+}(-K)^{q} n(p-1)}{\bar{t}}<\frac{\varepsilon}{24} .
\end{gathered}
$$

From (48) and (76), we see that

$$
\begin{aligned}
\mid \frac{1}{n} \int_{t}^{t+n} & \frac{s(\tau)}{\tau} \mathrm{d} \tau-\frac{1}{n} \int_{t}^{t+n} \frac{s(\tau)}{t} \mathrm{~d} \tau \mid \\
\quad= & \frac{1}{n}\left|\int_{t}^{t+n} s(\tau)\left(\frac{1}{\tau}-\frac{1}{t}\right) \mathrm{d} \tau\right| \\
\quad \leq & \frac{1}{n}\left|\int_{t}^{t+n} s(\tau) \mathrm{d} \tau\right| \frac{n}{t^{2}}<\frac{S n}{t^{2}}<\frac{\varepsilon}{24 t}, \quad t \geq \bar{t}
\end{aligned}
$$

from (55) and (77), we have

$$
\begin{aligned}
& \left|\frac{p-1}{n} \int_{t}^{t+n} \frac{\zeta(\tau)}{\tau} \mathrm{d} \tau-\frac{p-1}{n} \int_{t}^{t+n} \frac{\zeta(\tau)}{t} \mathrm{~d} \tau\right| \\
& \quad=\frac{p-1}{n}\left|\int_{t}^{t+n} \zeta(\tau)\left(\frac{1}{\tau}-\frac{1}{t}\right) \mathrm{d} \tau\right| \\
& \quad \leq \frac{p-1}{t^{2}} \int_{t}^{t+n}|\zeta(\tau)| \mathrm{d} \tau<\frac{-K n(p-1)}{t^{2}}<\frac{\varepsilon}{24 t}, \quad t \geq \bar{t},
\end{aligned}
$$

and, analogously, from (15), (55), and (78) it follows

$$
\begin{aligned}
& \left|\frac{p-1}{n} \int_{t}^{t+n} r(\tau) \frac{|\zeta(\tau)|^{q}}{\tau} \mathrm{d} \tau-\frac{p-1}{n} \int_{t}^{t+n} r(\tau) \frac{|\zeta(\tau)|^{q}}{t} \mathrm{~d} \tau\right| \\
& \quad=\frac{p-1}{n} \int_{t}^{t+n} r(\tau)|\zeta(\tau)|^{q}\left(\frac{1}{t}-\frac{1}{\tau}\right) \mathrm{d} \tau \\
& \quad \leq \frac{p-1}{t^{2}} \int_{t}^{t+n} r(\tau)|\zeta(\tau)|^{q} \mathrm{~d} \tau<\frac{r^{+}(-K)^{q} n(p-1)}{t^{2}} \\
& \quad<\frac{\varepsilon}{24 t}, \quad t \geq \bar{t} .
\end{aligned}
$$

For all $t \geq \bar{t}$, using (58), (75), (79), (80), and (81), we obtain

$\vartheta^{\prime}(t)$

$$
\begin{aligned}
= & \frac{1}{n} \int_{t}^{t+n} \zeta^{\prime}(\tau) \mathrm{d} \tau \\
= & \frac{1}{n} \int_{t}^{t+n} \frac{(p-1) \zeta(\tau)+s(\tau)+(p-1) r(\tau)|\zeta(\tau)|^{q}}{\tau} \mathrm{d} \tau \\
= & \frac{1}{n} \int_{t}^{t+n} \frac{s(\tau)}{\tau} \mathrm{d} \tau-\frac{q^{-p}}{t}+\frac{p-1}{n} \int_{t}^{t+n} \frac{\zeta(\tau)}{\tau} \mathrm{d} \tau \\
& +\frac{q^{-p}}{t}+\frac{p-1}{n} \int_{t}^{t+n} r(\tau) \frac{|\zeta(\tau)|^{q}}{\tau} \mathrm{d} \tau \\
> & \frac{1}{n} \int_{t}^{t+n} \frac{s(\tau)}{t} \mathrm{~d} \tau-\frac{q^{-p}}{t}+\frac{p-1}{n} \int_{t}^{t+n} \frac{\zeta(\tau)}{t} \mathrm{~d} \tau \\
& +\frac{q^{-p}}{t}+\frac{p-1}{n} \int_{t}^{t+n} r(\tau) \frac{|\zeta(\tau)|^{q}}{t} \mathrm{~d} \tau-\frac{\varepsilon}{8 t} \\
= & \frac{1}{t}\left[\frac{1}{n} \int_{t}^{t+n} s(\tau) \mathrm{d} \tau-q^{-p}+(p-1) \vartheta(t)+q^{-p}\right. \\
& \left.+\frac{p-1}{n} \int_{t}^{t+n} r(\tau)|\zeta(\tau)|^{q} \mathrm{~d} \tau-\frac{\varepsilon}{8}\right]>\frac{\varepsilon}{8 t} .
\end{aligned}
$$

Thus, it holds

$$
\vartheta(t)-\vartheta(\bar{t})=\int_{\bar{t}}^{t} \vartheta^{\prime}(\tau) \mathrm{d} \tau \geq \int_{\bar{t}}^{t} \frac{\varepsilon}{8 \tau} \mathrm{d} \tau, \quad t \geq \bar{t} .
$$

Since

$$
\lim _{t \rightarrow \infty} \int_{\bar{t}}^{t} \frac{\varepsilon}{8 \tau} \mathrm{d} \tau=\infty
$$

we obtain that $\lim _{t \rightarrow \infty} \vartheta(t)=\infty$. The contradiction with (62) proves the first implication.

In the nonoscillatory part of the proof, we consider $M(s)<q^{-p}$. Let $n \in \mathbb{N}$ and $\varepsilon>0$ satisfy

$$
\frac{1}{n} \int_{b}^{b+n} s(\tau) \mathrm{d} \tau-q^{-p}<-\varepsilon, \quad b \in[a, \infty) .
$$


Let us consider solution $\zeta$ of (17) given by $\zeta\left(T_{0}\right)=-1$ for some sufficiently large $T_{0} \geq a$. Since the right-hand side of (17) is continuous, the considered solution $\zeta$ can be defined on an interval $\left[T_{0}, T_{1}\right)$, where $T_{0}<T_{1} \leq \infty$. In addition, if $T_{1}<\infty$, we can assume that

$$
\limsup _{t \rightarrow T_{1}^{-}}|\zeta(t)|=\infty
$$

If $T_{1}=\infty$, then the considered solution of (17) satisfies the condition of Lemma 4 . It means that it suffices to find $B, C \in$ $\mathbb{R}$ for which

$$
B \leq \zeta(t) \leq C, \quad t \in\left[T_{0}, T_{1}\right)
$$

As in the oscillatory part of the proof (see (52)), we can prove that $\zeta(t)>K$ for some $K<-1$ and for all $t \in\left[T_{0}, T_{1}\right)$. Indeed, we can analogously show that the inequality $\zeta(t)<$ $-P-S T_{0}^{-1}$ cannot be valid for any $t \in\left[T_{0}, T_{1}\right)$, where $S$ is taken from (48) and $P$ from (53). We want to prove that $T_{1}=\infty$. On the contrary, let (86) be valid for some $T_{1} \in \mathbb{R}$. Particularly, solution $\zeta$ has to be positive on some interval $\left[T_{2}, T_{3}\right] \subset\left[T_{0}, T_{1}\right)$ in this case.

We denote

$$
\tilde{t}:=-\left(\frac{p}{p-1}\right)^{1-p}=-q^{1-p}
$$

and we compute

$$
\begin{aligned}
(p-1) \tilde{t}+q^{-p}+(p-1)|\tilde{t}|^{q} & =(1-p) q^{1-p}+p q^{-p} \\
& =q^{-p}[(1-p) q+p]=0 .
\end{aligned}
$$

We know that $\zeta$ is negative on an interval $\left[T_{0}, \widetilde{T}_{1}\right) \subseteq$ $\left[T_{0}, T_{1}\right)$. Let $\widetilde{T}_{1}$ have the property that $\zeta\left(\widetilde{T}_{1}\right)=0$. For all $t_{1}, t_{2} \in\left[T_{0}, \widetilde{T}_{1}\right], t_{1} \leq t_{2} \leq t_{1}+1$, we have (see (51))

$$
\begin{aligned}
\left|\zeta\left(t_{2}\right)-\zeta\left(t_{1}\right)\right|= & \left|\int_{t_{1}}^{t_{2}} \zeta^{\prime}(\tau) \mathrm{d} \tau\right| \\
\leq & (p-1) \int_{t_{1}}^{t_{2}} \frac{|\zeta(\tau)|+r^{+}|\zeta(\tau)|^{q}}{\tau} \mathrm{d} \tau \\
& +\left|\int_{t_{1}}^{t_{2}} \frac{s(\tau)}{\tau} \mathrm{d} \tau\right| \leq(p-1) \frac{|K|+r^{+}|K|^{q}}{t_{1}}+\frac{S}{t_{1}} \\
= & \frac{(p-1)\left[|K|+r^{+}|K|^{q}\right]+S}{t_{1}} .
\end{aligned}
$$

Thus, for general $t_{1}, t_{2} \in\left[T_{0}, \widetilde{T}_{1}\right]$ satisfying $t_{1} \leq t_{2} \leq t_{1}+2 n$, we have

$$
\left|\zeta\left(t_{2}\right)-\zeta\left(t_{1}\right)\right| \leq \frac{2 n\left([p-1]\left[|K|+r^{+}|K|^{q}\right]+S\right)}{t_{1}} .
$$

We can assume that $T_{0}$ is so large that

$$
\zeta(\tau)<0, \quad \tau \in[t, t+2 n] \subset\left[T_{0}, T_{1}\right),
$$

if $\zeta(t) \leq \tilde{t}$ (see $(88))$. Particularly $\left(\zeta\left(T_{0}\right)=-1<\widetilde{t}\right)$, we can define the function

$$
\vartheta(t):=\frac{1}{n} \int_{t}^{t+n} \zeta(\tau) \mathrm{d} \tau
$$

for all $t \in\left[T_{0}, T_{0}+n\right)$ and for all $t \geq T_{0}+n$ when $\zeta(t-n) \leq \tilde{t}$. Particularly, let $T_{0}$ be so large that $\vartheta\left(T_{0}\right)<\tilde{t}$.

We repeat that we assume the positivity of $\zeta$ which implies the inequality $\vartheta(t)>\tilde{t}$ for $t$ from some interval. The continuity of $\vartheta$ gives the existence of $\bar{t}>T_{0}$ such that

$$
\vartheta(\bar{t})=\tilde{t}, \quad \vartheta^{\prime}(\bar{t}) \geq 0 .
$$

From (91) it follows that, for any $\delta>0$, one can choose $T_{0}$ so large that

$$
\left|\zeta\left(t_{2}\right)-\zeta\left(t_{1}\right)\right|<\delta, \quad T_{0} \leq t_{1} \leq t_{2} \leq t_{1}+n \leq \widetilde{T}_{1} .
$$

Thus (see (93)), we can assume that

$$
|\vartheta(\bar{t})-\zeta(\tau)|<\delta, \quad \tau \in[\bar{t}, \bar{t}+n] .
$$

Consequently, let

$$
\left.|| \vartheta(\bar{t})\right|^{q}-|\zeta(\tau)|^{q} \mid<\frac{\varepsilon}{8(p-1)}, \quad \tau \in[\bar{t}, \bar{t}+n] .
$$

At the same time, we can assume that $n \in \mathbb{N}$ was chosen in such a way that it is valid

$$
\left|\frac{1}{n} \int_{b}^{b+n} r(\tau) \mathrm{d} \tau-1\right|<\min \left\{\frac{\varepsilon}{4(p-1)}, 1\right\}, \quad b \in[a, \infty) .
$$

Using (97) and (98), we have

$$
\begin{aligned}
& \mid\left[(p-1) \vartheta(\bar{t})+q^{-p}+\frac{p-1}{n} \int_{\bar{t}}^{\bar{t}+n} r(\tau)|\zeta(\tau)|^{q} \mathrm{~d} \tau\right] \\
& \quad-\left[(p-1) \vartheta(\bar{t})+q^{-p}+(p-1)|\vartheta(\bar{t})|^{q}\right] \mid \\
& =\left.(p-1)\left|\frac{1}{n} \int_{\bar{t}}^{\bar{t}+n} r(\tau)\right| \zeta(\tau)\right|^{q} \mathrm{~d} \tau-|\vartheta(\bar{t})|^{q} \\
& \quad+\frac{1}{n} \int_{\bar{t}}^{\bar{t}+n} r(\tau)|\vartheta(\bar{t})|^{q} \mathrm{~d} \tau-\frac{1}{n} \int_{\bar{t}}^{\bar{t}+n} r(\tau)|\vartheta(\bar{t})|^{q} \mathrm{~d} \tau \mid \\
& \leq\left.(p-1)\left|\frac{1}{n} \int_{\bar{t}}^{\bar{t}+n} r(\tau)\right| \zeta(\tau)\right|^{q} \mathrm{~d} \tau-\frac{1}{n} \int_{\bar{t}}^{\bar{t}+n} r(\tau)|\vartheta(\bar{t})|^{q} \mathrm{~d} \tau \mid \\
& \quad+\left.(p-1)|| \vartheta(\bar{t})\right|^{q}-\frac{1}{n} \int_{\bar{t}}^{\bar{t}+n} r(\tau)|\vartheta(\bar{t})|^{q} \mathrm{~d} \tau \mid \\
& \quad+(p-1)\left|\frac{1}{n} \int_{\bar{t}}^{\bar{t}+n} r(\tau)\left(|\zeta(\tau)|^{q}-|\vartheta(\bar{t})|^{q}\right) \mathrm{d} \tau\right| \\
& \quad+(p-1)\left|1-\frac{1}{n} \int_{\bar{t}}^{\bar{t}+n} r(\tau) \mathrm{d} \tau\right|<\frac{\varepsilon}{4}+\frac{\varepsilon}{4}=\frac{\varepsilon}{2} .
\end{aligned}
$$


Since (see (89), (94))

$$
\begin{aligned}
(p-1) & \vartheta(\bar{t})+q^{-p}+(p-1)|\vartheta(\bar{t})|^{q} \\
= & (p-1) \tilde{t}+q^{-p}+(p-1)|\tilde{t}|^{q}=0
\end{aligned}
$$

we have

$$
\left.\left|(p-1) \vartheta(\bar{t})+q^{-p}+\frac{p-1}{n} \int_{\bar{t}}^{\bar{t}+n} r(\tau)\right| \zeta(\tau)\right|^{q} \mathrm{~d} \tau \mid<\frac{\varepsilon}{2} .
$$

Let $T_{0}$ be so large that (see (48) and also (79))

$$
\left|\frac{1}{n} \int_{t}^{t+n} \frac{s(\tau)}{\tau} \mathrm{d} \tau-\frac{1}{n} \int_{t}^{t+n} \frac{s(\tau)}{t+n} \mathrm{~d} \tau\right|<\frac{\varepsilon}{8(t+n)}, \quad t \geq T_{0},
$$

and (see (15) together with (96) and also (81))

$$
\begin{aligned}
& \left|\frac{p-1}{n} \int_{\bar{t}}^{\bar{t}+n} \frac{r(\tau)|\zeta(\tau)|^{q}}{\tau} \mathrm{d} \tau-\frac{p-1}{n} \int_{\bar{t}}^{\bar{t}+n} \frac{r(\tau)|\zeta(\tau)|^{q}}{\bar{t}+n} \mathrm{~d} \tau\right| \\
& \quad<\frac{\varepsilon}{8(\bar{t}+n)} .
\end{aligned}
$$

Considering (85), (101), (102), and (103), we obtain

$\vartheta^{\prime}(\bar{t})$

$$
\begin{aligned}
= & \frac{1}{n} \int_{\bar{t}}^{\bar{t}+n} \zeta^{\prime}(\tau) \mathrm{d} \tau \\
= & \frac{1}{n} \int_{\bar{t}}^{\bar{t}+n} \frac{(p-1) \zeta(\tau)+s(\tau)+(p-1) r(\tau)|\zeta(\tau)|^{q}}{\tau} \mathrm{d} \tau \\
< & \frac{p-1}{n} \int_{\bar{t}}^{\bar{t}+n} \frac{\zeta(\tau)}{\bar{t}+n} \mathrm{~d} \tau+\frac{1}{n} \int_{\bar{t}}^{\bar{t}+n} \frac{s(\tau)}{\bar{t}+n} \mathrm{~d} \tau \\
& +\frac{p-1}{n} \int_{\bar{t}}^{\bar{t}+n} r(\tau) \frac{|\zeta(\tau)|^{q}}{\bar{t}+n} \mathrm{~d} \tau+\frac{\varepsilon}{4(\bar{t}+n)} \\
= & \frac{1}{\bar{t}+n}\left[(p-1) \vartheta(\bar{t})+q^{-p}+\frac{p-1}{n} \int_{\bar{t}}^{\bar{t}+n} r(\tau)|\zeta(\tau)|^{q} \mathrm{~d} \tau\right. \\
& \left.\quad+\frac{1}{n} \int_{\bar{t}}^{\bar{t}+n} s(\tau) \mathrm{d} \tau-q^{-p}+\frac{\varepsilon}{4}\right]<-\frac{\varepsilon}{4(\bar{t}+n)} .
\end{aligned}
$$

This contradiction (see (94)) means that (87) is true for $B=K$ and $C=0$. Since (86) cannot be valid for any $T_{1}<\infty$, the considered solution $\zeta$ exists on interval $\left[T_{0}, \infty\right)$. We repeat that the nonoscillation of (14) actually follows from Lemma 4.

The following theorem is a version of Theorem 8 which is ready for applications to the half-linear equations written in the form common in the literature.
Theorem 9. Let $c: \mathbb{R}_{a} \rightarrow \mathbb{R}$ be a continuous function, for which mean value $M\left(c^{1-q}\right)$ exists and for which it holds

$$
0<\inf _{t \in \mathbb{R}_{a}} c(t) \leq \sup _{t \in \mathbb{R}_{a}} c(t)<\infty,
$$

and let $d: \mathbb{R}_{a} \rightarrow \mathbb{R}$ be a continuous function having mean value $M(d)$. Let

$$
\Gamma:=q^{-p}\left[M\left(c^{1-q}\right)\right]^{1-p}=q^{-p}\left[\lim _{t \rightarrow \infty} \frac{1}{t} \int_{a}^{a+t} c^{1-q}(\tau) d \tau\right]^{1-p} .
$$

\section{Consider the equation}

$$
\left[c(t) \Phi\left(x^{\prime}\right)\right]^{\prime}+\frac{d(t)}{t^{p}} \Phi(x)=0, \quad \Phi(x)=|x|^{p-1} \operatorname{sgn} x .
$$

Equation (107) is oscillatory if $M(d)>\Gamma$ and nonoscillatory if $M(d)<\Gamma$.

Proof. Let $M(d)>0$. Equation(107) can be rewritten into the form

$$
\left[\left[c^{1-q}(t)\right]^{-p / q} \Phi\left(x^{\prime}\right)\right]^{\prime}+\frac{d(t)}{t^{p}} \Phi(x)=0 ;
$$

that is,

$$
\left[\left[\frac{c^{1-q}(t)}{M\left(c^{1-q}\right)}\right]^{-p / q} \Phi\left(x^{\prime}\right)\right]^{\prime}+\frac{\left[M\left(c^{1-q}\right)\right]^{p / q} d(t)}{t^{p}} \Phi(x)=0 .
$$

Equation (109) has the form of (14) for

$$
r(t)=\frac{c^{1-q}(t)}{M\left(c^{1-q}\right)}, \quad s(t)=\left[M\left(c^{1-q}\right)\right]^{p / q} d(t), \quad t \geq a .
$$

Note that $M(r)=1$ and $M(s)>0$ and that (15) follows from (105). Thus (see Theorem 8), (107) is oscillatory for

$$
\begin{array}{r}
M(s)=\left[M\left(c^{1-q}\right)\right]^{p / q} M(d)=\left[M\left(c^{1-q}\right)\right]^{p-1} M(d)>q^{-p}, \\
\text { i.e., } M(d)>\Gamma,
\end{array}
$$

and nonoscillatory if the opposite inequality $M(d)<\Gamma$ holds.

It remains to consider the case when $M(d) \leq 0$. Of course, there exists $k>0$ such that $0<M(d+k)=M(d)+k<\Gamma$. We know that the equation

$$
\left[c(t) \Phi\left(x^{\prime}\right)\right]^{\prime}+\frac{d(t)+k}{t^{p}} \Phi(x)=0
$$

is nonoscillatory. Now it suffices to use Theorem 2 (ii).

Remark 10. For reader's convenience, we consider (107) (instead of (14)) in Theorem 9. The form of (107) shows how the presented result improves the known ones (see Section 1). Particularly, we get new results in two important cases, when function $s$ changes sign and when it is unbounded. For details, we refer to our previous paper [18]. 
Remark 11. For $M(d)=\Gamma$, it is not possible to decide whether (107) is oscillatory or nonoscillatory for general functions $c, d$ satisfying the conditions from the statement of Theorem 9. It follows, for example, from the main results of $[13,16]$. One of the most studied classes of functions which have mean values is formed by almost periodic functions. Based on the constructions from [26], it is conjectured in [18] that the case $M(d)=\Gamma$ is not generally solvable (in the sense whether it is oscillatory or nonoscillatory) even for almost periodic coefficients of (107). It means that there exist almost periodic functions $c$, $d$ such that $M(d)=\Gamma$ and (107) is oscillatory. At the same time, there exist different almost periodic functions $c, d$ satisfying $M(d)=\Gamma$ with the property that (107) is nonoscillatory. We add that the case of periodic functions $c, d$ was proved to be nonoscillatory (see again $[13,16]$ ).

To illustrate Theorem 9, we mention at least two examples.

Example 12. For $A>1 / 2, B, C>0$, and $q=3$, let us consider the equation

$$
\begin{aligned}
& {\left[\frac{\Phi\left(x^{\prime}\right)}{A+\cos (B t) \sin (B t)}\right]^{\prime}} \\
& \quad+\frac{\arcsin (\cos t)+[\cos (C t) \sin (C t)]^{2}}{\sqrt{t^{3}+t^{2}+t+1}} \Phi(x)=0 .
\end{aligned}
$$

Equation (113) has the form of (107) for

$$
\begin{gathered}
c(t)=\frac{1}{A+\cos (B t) \sin (B t)} \\
d(t)=\frac{\arcsin (\cos t)+[\cos (C t) \sin (C t)]^{2}}{\sqrt{1+(1 / t)+\left(1 / t^{2}\right)+\left(1 / t^{3}\right)}}
\end{gathered}
$$

It can be directly verified that

$$
M\left(c^{1-q}\right)=M\left([\cos (B t) \sin (B t)+A]^{2}\right)=\frac{1+8 A^{2}}{8}
$$

and that

$$
M(d)=M\left([\cos (C t) \sin (C t)]^{2}\right)=\frac{1}{8} .
$$

Hence, (113) is oscillatory if $2^{9}<3^{3}\left(1+8 A^{2}\right)$ and nonoscillatory if $2^{9}>3^{3}\left(1+8 A^{2}\right)$; that is, (113) is oscillatory for $A \in(\kappa, \infty)$ and nonoscillatory for $A \in(1 / 2, \kappa)$, where $\kappa=\sqrt{\left(8^{3} / 3^{3}-1\right) / 8} \doteq 1.498455995$. Since $d$ is oscillatory, the other related results in the literature give no conclusion for (107).
Example 13. Let $K \in \mathbb{R}$ and $k \neq 0$ be arbitrarily given. We define the function $d: \mathbb{R}_{1} \rightarrow \mathbb{R}$ by

$$
\begin{aligned}
& d(t):=K+k(t-n) 3^{n}, \quad t \in\left[n, n+\frac{1}{2^{n}}\right), n \in \mathbb{N} ; \\
& d(t):=K+k\left(n+\frac{2}{2^{n}}-t\right) 3^{n}, \quad t \in\left[n+\frac{1}{2^{n}}, n+\frac{2}{2^{n}}\right), \\
& \left.d(t):=K, \quad t \in \mathbb{N} ; n+\frac{2}{2^{n}}, n+1\right), n \in \mathbb{N} .
\end{aligned}
$$

It is seen that $\limsup _{t \rightarrow \infty}|d(t)|=\infty$ and that $M(d)=K$. Analogously, for $L>0$ and $l>-L$, we define $c: \mathbb{R}_{1} \rightarrow \mathbb{R}$ as

$$
\begin{aligned}
& c(t):=L+l(t-n) 2 n, \quad t \in\left[n, n+\frac{1}{2 n}\right), n \in \mathbb{N} \\
& c(t):=L+l\left(n+\frac{1}{n}-t\right) 2 n, \quad t \in\left[n+\frac{1}{2 n}, n+\frac{1}{n}\right), n \in \mathbb{N} \\
& c(t):=L, \quad t \in\left[n+\frac{1}{n}, n+1\right), n \in \mathbb{N} .
\end{aligned}
$$

We have (105) and $M\left(c^{1-q}\right)=L^{1-q}$. Thus, (107) for the above given functions $c, d$ is oscillatory if $K>q^{-p} L$ and nonoscillatory if $K<q^{-p} L$. Since $d$ is not bounded, the other related results in the literature give no conclusion for this equation.

Theorem 9 gives a new result for linear equations as well. Therefore, we mention the following corollary.

Corollary 14. Let $r, s: \mathbb{R}_{a} \rightarrow \mathbb{R}$ be continuous functions having mean values $M(r), M(s)$ and let (15) hold. Then, the equation

$$
\left[\frac{x^{\prime}}{r(t)}\right]^{\prime}+\frac{s(t)}{t^{2}} x=0
$$

is oscillatory if $4 M(r) M(s)>1$ and nonoscillatory if $4 M(r) M(s)<1$.

Example 15. Using Corollary 14, we can decide about the oscillation and nonoscillation of several equations. Let $a, b \in$ $\mathbb{R}$ and $m, n \in \mathbb{N}$ be relative prime. Let $f, g: \mathbb{R}_{1} \rightarrow \mathbb{R}$ be arbitrary positive continuous functions with the property that

$$
\lim _{t \rightarrow \infty} f(t)=1, \quad \lim _{t \rightarrow \infty} \frac{g(t)}{t^{2}}=1
$$


The equations

$$
\begin{aligned}
& {\left[f(t) x^{\prime}\right]^{\prime}+\gamma \frac{|\sin (\sqrt{m} t+a)|+\sin (\sqrt{n} t+b)}{g(t)} x=0,} \\
& {\left[f(t) x^{\prime}\right]^{\prime}+\gamma \frac{|\cos (\sqrt{m} t+a)|+\sin (\sqrt{n} t+b)}{g(t)} x=0,} \\
& {\left[f(t) x^{\prime}\right]^{\prime}+\gamma \frac{|\sin (\sqrt{m} t+a)|+\cos (\sqrt{n} t+b)}{g(t)} x=0,} \\
& {\left[f(t) x^{\prime}\right]^{\prime}+\gamma \frac{|\cos (\sqrt{m} t+a)|+\cos (\sqrt{n} t+b)}{g(t)} x=0}
\end{aligned}
$$

are oscillatory for $8 \gamma>\pi$ and nonoscillatory for $8 \gamma<\pi$. Note that this simple criterion does not follow from known ones.

Now we consider the case of negative coefficient $r$.

Corollary 16. Let $r, s: \mathbb{R}_{a} \rightarrow \mathbb{R}$ be continuous functions such that function s has mean value $M(s)$ and that there exists mean value $R:=M\left(|r|^{1-q}\right)$. Let

$$
-\infty<\inf _{t \in \mathbb{R}_{a}} r(t) \leq \sup _{t \in \mathbb{R}_{a}} r(t)<0
$$

be fulfilled. The equation

$$
\left[r(t) \Phi\left(x^{\prime}\right)\right]^{\prime}+\frac{s(t)}{t^{p}} \Phi(x)=0
$$

is oscillatory if $M(s)<-q^{-p} R^{1-p}$ and nonoscillatory if $M(s)>$ $-q^{-p} R^{1-p}$.

Proof. To prove the corollary, it suffices to use Theorem 9 and the fact that the solution space of half-linear equations is homogeneous.

One can prove a number of consequences using different types of comparison theorems. For example, applying Theorem 9 and [10, Theorem 2.3.1], we immediately obtain the next new result.

Corollary 17. Let $r, s: \mathbb{R}_{a} \rightarrow \mathbb{R}$ be continuous functions having mean values $M(r), M(s)$. Let (15) hold and let

$$
\Gamma:=q^{-p}[M(r)]^{1-p}=q^{-p}\left[\lim _{t \rightarrow \infty} \frac{1}{t} \int_{a}^{a+t} r(\tau) d \tau\right]^{1-p} .
$$

Let us consider the equation

$$
\left[r^{-p / q}(t) \Phi\left(x^{\prime}\right)\right]^{\prime}+y(t) \Phi(x)=0,
$$

where $y: \mathbb{R}_{a} \rightarrow \mathbb{R}$ is a continuous function satisfying $\int_{a}^{\infty} y(\tau) d \tau<\infty$.

(i) If there exists $t_{0} \geq a$ for which

$$
\left|\int_{t}^{\infty} \frac{s(\tau)}{\tau^{p}} d \tau\right| \leq \int_{t}^{\infty} y(\tau) d \tau, \quad t \geq t_{0}
$$

and if $M(s)>\Gamma$, then (125) is oscillatory. (ii) If there exists $t_{0} \geq a$ for which

$$
\int_{t}^{\infty} \frac{s(\tau)}{\tau^{p}} d \tau \geq\left|\int_{t}^{\infty} y(\tau) d \tau\right|, \quad t \geq t_{0}
$$

and if $M(s)<\Gamma$, then (125) is nonoscillatory.

In the following example, we demonstrate that our result is applicable even if the mean value of the second coefficient does not exist.

Example 18. Let $p>1$ and $X, Y, Z_{1}, Z_{2}>0$. Putting

$$
\begin{aligned}
& s(t):=X+Y\left(t-2^{n}\right) 2^{n}, \quad t \in\left[2^{n}, 2^{n}+\frac{1}{4}\right), \\
& n \in \mathbb{N} ; \\
& s(t):=X+Y\left(2^{n}+\frac{1}{2}-t\right) 2^{n}, \quad t \in\left[2^{n}+\frac{1}{4}, 2^{n}+\frac{1}{2}\right), \\
& s(t):=X-Y\left(t-2^{n}-\frac{1}{2}\right) 2^{n}, \quad t \in\left[2^{n}+\frac{1}{2}, 2^{n}+\frac{3}{4}\right), \\
& s(t):=X-Y\left(2^{n}+1-t\right) 2^{n}, \quad t \in\left[2^{n}+\frac{3}{4}, 2^{n}+1\right], \\
& \begin{array}{c}
n \in \mathbb{N} ; \\
s(t):=X, \quad t \in \mathbb{R}_{2} \backslash \bigcup_{n \in \mathbb{N}}\left[2^{n}, 2^{n}+1\right], \\
n \in \mathbb{N} ;
\end{array}
\end{aligned}
$$

we define the continuous function $s: \mathbb{R}_{2} \rightarrow \mathbb{R}$. Evidently, mean value $M(s)$ does not exist. Since

$$
\begin{aligned}
0 & <\int_{2^{n}}^{2^{n+1}} \frac{s(\tau)-X}{\tau^{p}} \mathrm{~d} \tau \\
& =\int_{2^{n}}^{2^{n+1}} \frac{s(\tau)-X}{\tau^{p}} \mathrm{~d} \tau<\frac{Y}{16}\left[\frac{2^{n}}{\left(2^{n}\right)^{p}}-\frac{2^{n}}{\left(2^{n}+1\right)^{p}}\right] \\
& =\frac{Y \cdot 2^{n}}{16}\left[\frac{\left(2^{n}+1\right)^{p}-\left(2^{n}\right)^{p}}{\left(2^{n}+1\right)^{p}\left(2^{n}\right)^{p}}\right]<\frac{Y}{16} \cdot \frac{1}{\left(2^{n}\right)^{p-1}}, \quad n \in \mathbb{N}
\end{aligned}
$$

we have

$$
0<\int_{2}^{\infty} \frac{s(\tau)}{\tau^{p}} \mathrm{~d} \tau<\frac{Y}{16} \sum_{n=1}^{\infty} \frac{1}{\left(2^{p-1}\right)^{n}}+\int_{2}^{\infty} \frac{X}{\tau^{p}} \mathrm{~d} \tau<\infty .
$$

Considering

$$
\lim _{n \rightarrow \infty} \frac{\left(\left(2^{n}+1\right)^{p}-\left(2^{n}\right)^{p}\right) /\left(\left(2^{n}+1\right)^{p}\left(2^{n}\right)^{p}\right)}{1 / 2^{[n+1]} p}=0
$$

and (129), there exists a positive continuous function $h$ : $\mathbb{R}_{2} \rightarrow \mathbb{R}$ with the property that

$$
\int_{t}^{2^{n+1}} \frac{h(\tau)}{\tau^{p}} \mathrm{~d} \tau>\left|\int_{t}^{2^{n+1}} \frac{s(\tau)-X}{\tau^{p}} \mathrm{~d} \tau\right|, \quad t \in\left[2^{n}, 2^{n+1}\right), n \in \mathbb{N}
$$


and that $\lim _{t \rightarrow \infty} h(t)=0$. Using (132), we obtain

$$
\int_{t}^{\infty} \frac{X-h(\tau)}{\tau^{p}} \mathrm{~d} \tau<\int_{t}^{\infty} \frac{s(\tau)}{\tau^{p}} \mathrm{~d} \tau<\int_{t}^{\infty} \frac{X+h(\tau)}{\tau^{p}} \mathrm{~d} \tau, \quad t \geq 2 .
$$

In addition, $M(X+h)=M(X-h)=X$ and

$$
\int_{2}^{\infty} \frac{h(\tau)}{\tau^{p}} \mathrm{~d} \tau<\infty
$$

It is also seen that

$$
\int_{t}^{\infty} \frac{X-h(\tau)}{\tau^{p}} \mathrm{~d} \tau>0
$$

for all sufficiently large $t$. The equations

$$
\begin{aligned}
& {\left[\left(\left|\sin \left[Z_{1} t\right]\right|+\left|\cos \left[Z_{1} t\right]\right|+\left|\sin \left[Z_{2} t\right]\right|+\left|\cos \left[Z_{2} t\right]\right|\right)^{-p / q}\right.} \\
& \left.\quad \times \Phi\left(x^{\prime}\right)\right]^{\prime}+\frac{X-h(t)}{t^{p}} \Phi(x)=0, \\
& {\left[\left(\left|\sin \left[Z_{1} t\right]\right|+\left|\cos \left[Z_{1} t\right]\right|+\left|\sin \left[Z_{2} t\right]\right|+\left|\cos \left[Z_{2} t\right]\right|\right)^{-p / q}\right.} \\
& \left.\quad \times \Phi\left(x^{\prime}\right)\right]^{\prime}+\frac{X+h(t)}{t^{p}} \Phi(x)=0
\end{aligned}
$$

are oscillatory for $X>q^{-p}(\pi / 8)^{p-1}$ and nonoscillatory for $X<q^{-p}(\pi / 8)^{p-1}$ (see Theorem 9). Indeed,

$$
\begin{aligned}
M( & {\left[\left|\sin \left(Z_{1} t\right)\right|+\left|\cos \left(Z_{1} t\right)\right|+\left|\sin \left(Z_{2} t\right)\right|\right.} \\
+ & \left.\left.\left|\cos \left(Z_{2} t\right)\right|\right]^{(p(q-1)) / q}\right)=\frac{8}{\pi} .
\end{aligned}
$$

Therefore (see Corollary 17 together with (130), (133), (134), and (135)), we know that the equation

$$
\begin{aligned}
& {\left[\left(\left|\sin \left[Z_{1} t\right]\right|+\left|\cos \left[Z_{1} t\right]\right|+\left|\sin \left[Z_{2} t\right]\right|+\left|\cos \left[Z_{2} t\right]\right|\right)^{-p / q}\right.} \\
& \left.\quad \times \Phi\left(x^{\prime}\right)\right]^{\prime}+\frac{s(t)}{t^{p}} \Phi(x)=0
\end{aligned}
$$

is oscillatory if $X>q^{-p}(\pi / 8)^{p-1}$ and nonoscillatory if $X<$ $q^{-p}(\pi / 8)^{p-1}$.

\section{An Application}

In this section, we use our main result to derive a theorem related to elliptic partial differential equations with $p$ Laplacian and the power-type nonlinearity

$$
\operatorname{div}\left(A(x)\|\nabla u\|^{p-2} \nabla u\right)+C(x) \Phi(u)=0,
$$

where $x=\left(x_{i}\right)_{i=1}^{n} \in \mathbb{R}^{n}, A$ is an elliptic $n \times n$ matrix function with differentiable components, and $C$ is a Hölder continuous function. As a solution of (139) in $\Omega \subseteq \mathbb{R}^{n}$, we understand a differentiable function $u$ such that $A(x)\|\nabla u(x)\|^{p-2} \nabla u(x)$ is also differentiable and $u$ satisfies (139) in $\Omega$.

The following notation is used. We consider the usual Euclidean norm

$$
\|\vec{b}\|=\left(\sum_{i=1}^{n} b_{i}^{2}\right)^{1 / 2}
$$

and the induced matrix norm

$$
\|A\|=\sup _{\|\vec{b}\| \neq 0} \frac{\|A \vec{b}\|}{\|\vec{b}\|}
$$

and $\lambda_{\min }(x), \lambda_{\max }(x)$ stand for the smallest and largest eigenvalues of matrix $A(x)$, respectively. From the fact that $A(x)$ is positive definite, it follows that $\|A(x)\|=\lambda_{\text {max }}(x)$.

Denote $\Omega\left(r_{0}\right):=\left\{x \in \mathbb{R}^{n}:\|x\| \geq r_{0}\right\}$. We say that a solution $u$ of (139) is oscillatory if it has a zero in $\Omega(r)$ for every $r \geq r_{0}$. Equation (139) is said to be oscillatory if every solution of this equation is oscillatory. Otherwise, (139) is said to be nonoscillatory.

In general, we distinguish two types of oscillation in the theory of (139), namely, the (weak) oscillation defined in the previous paragraph and the so-called (strong) nodal oscillation, which is based on nodal domains (i.e., bounded domains such that the equation possesses a nontrivial solution which vanishes on the boundary of this domain). Concerning this concept of oscillation, (139) is said to be nodally oscillatory if every solution has a nodal domain outside of any ball in $\mathbb{R}^{n}$ and to be nodally nonoscillatory in the opposite case. It is known that the nodal oscillation implies oscillation. The opposite implication has been proved only in the linear case $p=2$ (see [27]) and remains an open question in the halflinear multidimensional case. We also refer to [28] which relates the weak oscillation of linear PDEs (and nodal oscillation) with the finiteness of negative spectrum of the Laplace operator, which is of interest in physical applications.

In $[29,30]$, there is proved a theorem which allows to deduce the oscillation of certain half-linear partial differential equations from the oscillation of ordinary differential equations if $A(x)$ is either the identity matrix or a scalar multiple of the identity matrix. This theorem has been later extended in [31] (see also [32]) as follows.

Theorem 19. Let us define the functions

$$
\begin{array}{ll}
\tilde{r}(t):= \begin{cases}\int_{\|x\|=t}\left(\frac{\lambda_{\max }(x)}{\lambda_{\min }(x)}\right)^{p-1} \lambda_{\max }(x) d S & \text { for } p>2 ; \\
\int_{\|x\|=t} \lambda_{\max }(x) d S & \text { for } 1<p \leq 2,\end{cases} \\
\widetilde{c}(t):=\int_{\|x\|=t} C(x) d S . &
\end{array}
$$

If the equation

$$
\left(\widetilde{r}(t) \Phi\left(u^{\prime}\right)\right)^{\prime}+\widetilde{c}(t) \Phi(u)=0
$$

is oscillatory, then (139) is oscillatory as well. 
We can easily apply the oscillation part of Theorems 9 and 19 to obtain the following result.

Theorem 20. Let $\widetilde{r}(t)$ and $\widetilde{c}(t)$ be defined by (142). Suppose that

$$
0<\liminf _{t \rightarrow \infty} \widetilde{r}(t) \leq \limsup _{t \rightarrow \infty} \widetilde{r}(t)<\infty
$$

and that $M\left(\widetilde{r}^{1-q}\right)$ and $M\left(\widetilde{c}(t) t^{p}\right)$ exist. If

$$
M\left(\widetilde{c}(t) t^{p}\right)>q^{-p}\left[M\left(\widetilde{r}^{1-q}\right)\right]^{1-p},
$$

then (139) is oscillatory.

Remark 21. Note that, in contrast to Theorem 9, we lack the nonoscillation part in Theorems 19 and 20 because there is a principal problem with nonoscillation criteria for partial differential equations via the Riccati method. A detailed discussion related to the relationship of the Riccati equation and the nonoscillation of second-order equations (in the multidimensional case) can be found in [33].

\section{Conflict of Interests}

The authors declare that there is no conflict of interests regarding the publication of this paper.

\section{Acknowledgments}

Petr Hasil and Robert Mařík are supported by Czech Science Foundation under Grant P201/10/1032. Michal Veselý is supported by the project "Employment of Best Young Scientists for International Cooperation Empowerment" (CZ.1.07/2.3.00/30.0037) cofinanced from European Social Fund and the state budget of the Czech Republic.

\section{References}

[1] A. Kneser, "Untersuchungen über die reellen Nullstellen der Integrale linearer Differentialgleichungen," Mathematische Annalen, vol. 42, no. 3, pp. 409-435, 1893.

[2] F. Gesztesy and M. Ünal, "Perturbative oscillation criteria and Hardy-type inequalities," Mathematische Nachrichten, vol. 189, pp. 121-144, 1998.

[3] K. M. Schmidt, "Oscillation of the perturbed Hill equation and the lower spectrum of radially periodic Schrödinger operators in the plane," Proceedings of the American Mathematical Society, vol. 127, no. 8, pp. 2367-2374, 1999.

[4] H. Krüger, "On perturbations of quasiperiodic Schrödinger operators," Journal of Differential Equations, vol. 249, no. 6, pp. 1305-1321, 2010.

[5] H. Krüger and G. Teschl, "Effective Prüfer angles and relative oscillation criteria," Journal of Differential Equations, vol. 245, no. 12, pp. 3823-3848, 2008.

[6] H. Krüger and G. Teschl, "Relative oscillation theory for SturmLiouville operators extended," Journal of Functional Analysis, vol. 254, no. 6, pp. 1702-1720, 2008.

[7] H. Krüger and G. Teschl, "Relative oscillation theory, weighted zeros of the Wronskian, and the spectral shift function," Communications in Mathematical Physics, vol. 287, no. 2, pp. 613640, 2009.
[8] K. M. Schmidt, "Critical coupling constants and eigenvalue asymptotics of perturbed periodic Sturm-Liouville operators," Communications in Mathematical Physics, vol. 211, no. 2, pp. 465-485, 2000.

[9] R. P. Agarwal, S. R. Grace, and D. O'Regan, Oscillation Theory for Second Order Linear, Half-Linear, Superlinear and Sublinear Dynamic Equations, Kluwer Academic Publishers, Dordrecht, The Netherlands, 2002.

[10] O. Došlý and P. Řehák, Half-Linear Differential Equations, Elsevier, Amsterdam, The Netherlands, 2005.

[11] Á. Elbert, "Oscillation and nonoscillation theorems for some nonlinear ordinary differential equations," in Ordinary and Partial Differential Equations (Dundee, 1982), vol. 1964 of Lecture Notes in Mathematics, pp. 187-212, Springer, Berlin, Germany, 1982.

[12] Á. Elbert, "Asymptotic behaviour of autonomous half-linear differential systems on the plane," Studia Scientiarum Mathematicarum Hungarica, vol. 19, no. 2-4, pp. 447-464, 1984.

[13] O. Došlý and H. Funková, "Euler type half-linear differential equations with periodic coefficients," Abstract and Applied Analysis, vol. 2013, Article ID 714263, 6 pages, 2013.

[14] O. Došlý and H. Funková, "Perturbations of half-linear Euler differential equation and transformations of modified Riccati equation," Abstract and Applied Analysis, vol. 2012, Article ID 738472, 19 pages, 2012.

[15] O. Došlý and H. Haladová, "Half-linear Euler differential equations in the critical case," Tatra Mountains Mathematical Publications, vol. 48, no. 1, pp. 41-49, 2011.

[16] O. Došlý and P. Hasil, "Critical oscillation constant for halflinear differential equations with periodic coefficients," Annali di Matematica Pura ed Applicata, vol. 190, no. 3, pp. 395-408, 2011.

[17] P. Hasil, "Conditional oscillation of half-linear differential equations with periodic coefficients," Archivum Mathematicum, vol. 44, no. 2, pp. 119-131, 2008.

[18] P. Hasil and M. Veselý, "Oscillation of half-linear differential equations with asymptotically almost periodic coefficients," Advances in Difference Equations, vol. 2013, article 122, 2013.

[19] P. Hasil and M. Veselý, "Critical oscillation constant for difference equations with almost periodic coefficients," Abstract and Applied Analysis, vol. 2012, Article ID 471435, 19 pages, 2012.

[20] M. Veselý and P. Hasil, "Oscillation and nonoscillation of asymptotically almost periodic half-linear difference equations," Abstract and Applied Analysis, vol. 2013, Article ID 432936, 12 pages, 2013.

[21] O. Došlý and S. Fišnarová, "Linearized Riccati technique and (non-)oscillation criteria for half-linear difference equations," Advances in Difference Equations, vol. 2008, Article ID 438130, pp. 1-18, 2008.

[22] N. Yamaoka, "Oscillation criteria for second-order nonlinear difference equations of Euler type," Advances in Difference Equations, vol. 2012, article 218, 14 pages, 2012.

[23] P. Řehák, "A critical oscillation constant as a variable of time scales for half-linear dynamic equations," Mathematica Slovaca, vol. 60, no. 2, pp. 237-256, 2010.

[24] P. Řehák, "New results on critical oscillation constants depending on a graininess," Dynamic Systems and Applications, vol. 19, no. 2, pp. 271-287, 2010.

[25] P. Řehák, "On certain comparison theorems for half-linear dynamic equations on time scales," Abstract and Applied Analysis, vol. 2004, no. 7, pp. 551-565, 2004. 
[26] M. Veselý, "Construction of almost periodic functions with given properties," Electronic Journal of Differential Equations, vol. 2011, no. 29, pp. 1-25, 2011.

[27] W. F. Moss and J. Piepenbrink, "Positive solutions of elliptic equations," Pacific Journal of Mathematics, vol. 75, no. 1, pp. 219$226,1978$.

[28] U. W. Schmincke, "The lower spectrum of Schrödinger operators," Archive for Rational Mechanics and Analysis, vol. 75, no. 2, pp. 147-155, 1989.

[29] O. Došlý and R. Mařík, "Nonexistence of positive solutions of PDE's with p-Laplacian," Acta Mathematica Hungarica, vol. 90, no. 1-2, pp. 89-107, 2001.

[30] T. Kusano, J. Jaroš, and N. Yoshida, "A Picone-type identity and Sturmian comparison and oscillation theorems for a class of half-linear partial differential equations of second order," Nonlinear Analysis: Theory, Methods and Applications, vol. 40, no. 1-8, pp. 381-395, 2000.

[31] S. Fišnarová and R. Mařík, "Generalized Picone and Riccati inequalities for half-linear differential operators with arbitrary elliptic matrices," Electronic Journal of Differential Equations, vol. 2010, no. 111, pp. 1-13, 2010.

[32] R. Mařík, "Ordinary differential equations in the oscillation theory of partial half-linear differential equation," Journal of Mathematical Analysis and Applications, vol. 338, no. 1, pp. 194208, 2008.

[33] O. Došlý, S. Fišnarová, and R. Mařík, "Comparison theorems for Riccati inequalities arising in the theory of PDE's with $p$ Laplacian," Electronic Journal of Differential Equations, vol. 2011, no. 53, pp. 1-9, 2011. 


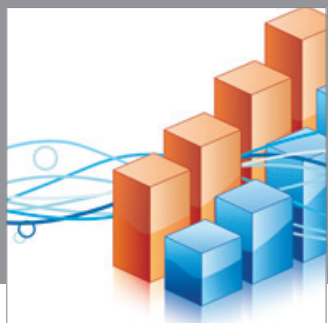

Advances in

Operations Research

mansans

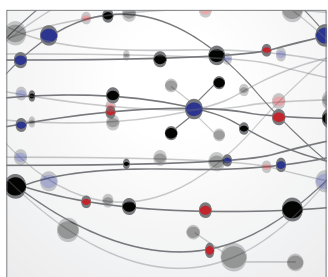

The Scientific World Journal
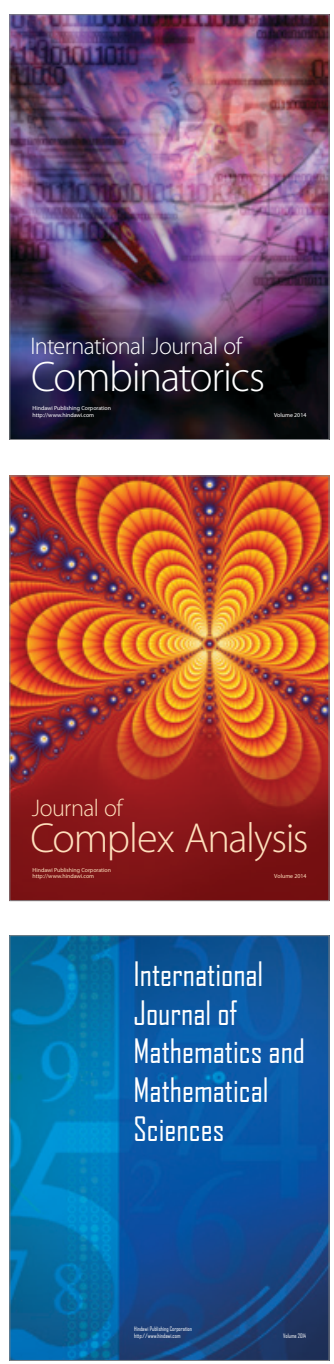
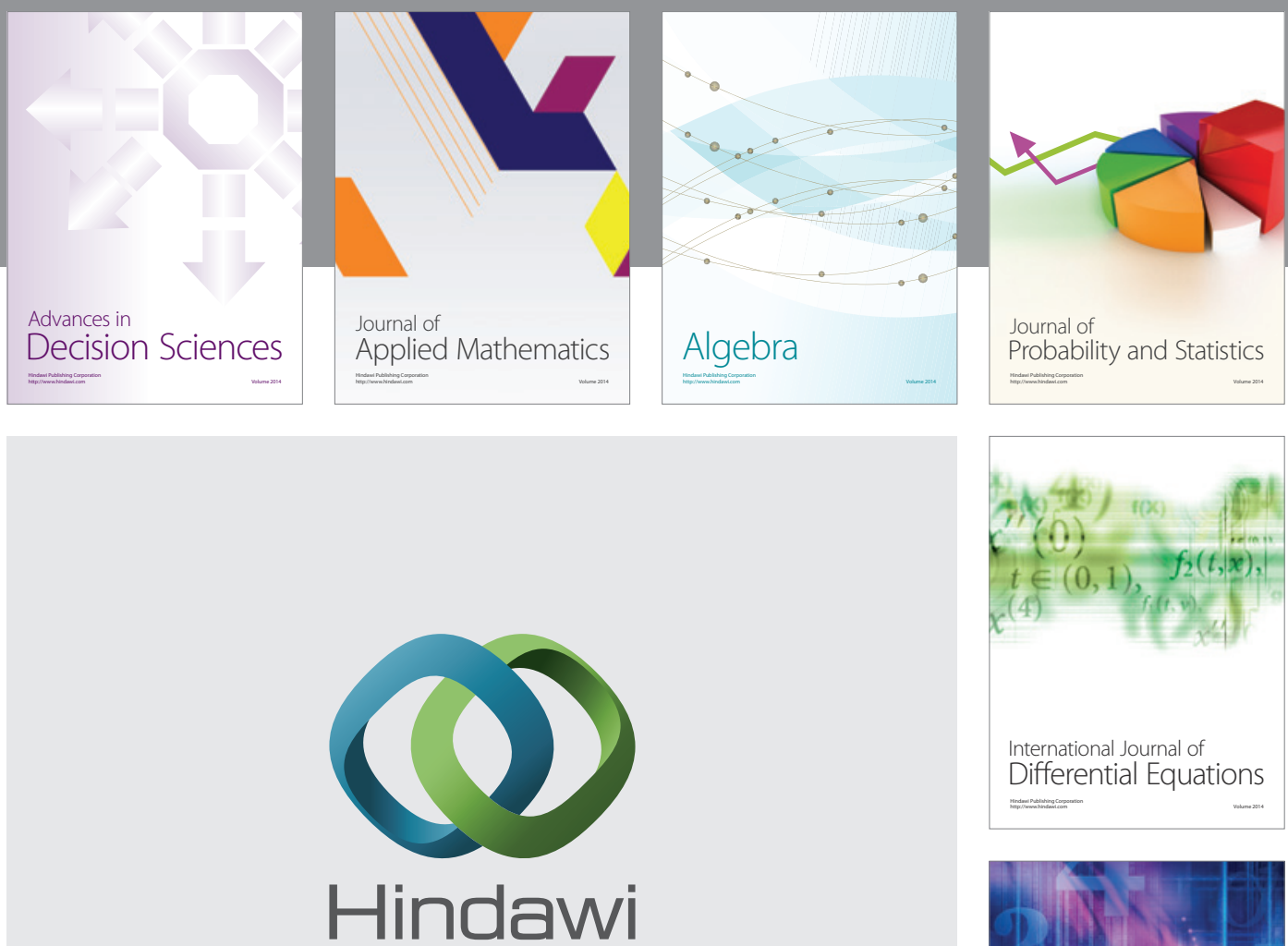

Submit your manuscripts at http://www.hindawi.com
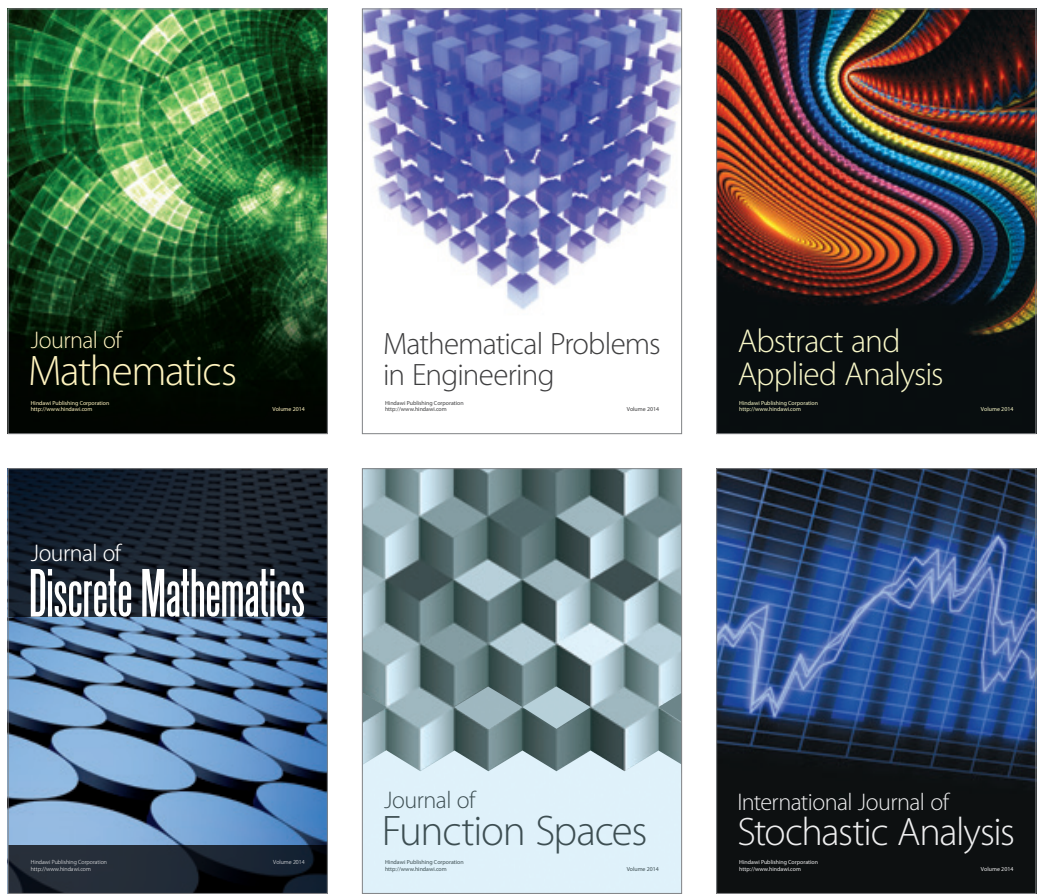

Journal of

Function Spaces

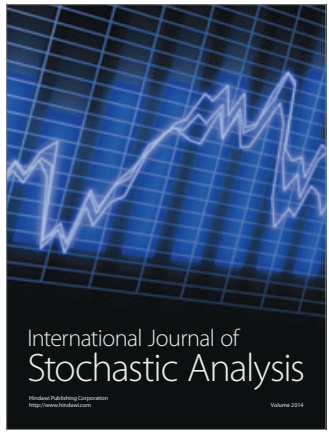

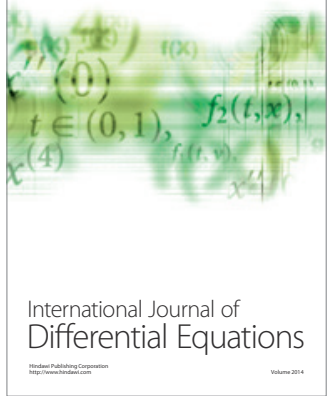
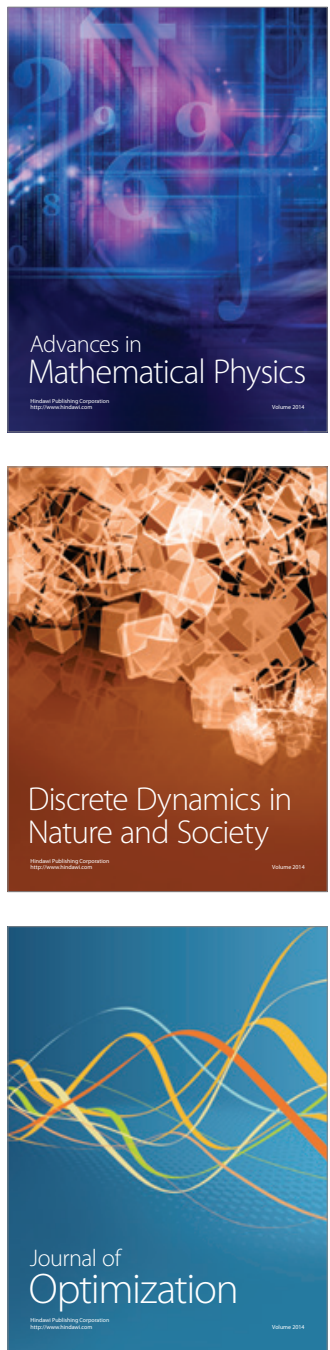